\title{
Epistemicity and Deixis: Perspectives from Central Alaskan Yup'ik
}

\author{
YUKI-SHIGE TAMURA \\ Shiga University
}

\section{Introduction}

The demonstrative system of Eskimo languages is known to show one of the most complicated systems in deictic reference among languages worldwide (Fotescue 1988, Rukeyzer 2005). The aim of this paper is, employing Central Alaskan Yup'ik (an Eskimo language) as an example, to characterize one vital portion of the complicated system from the perspective of Cognitive Linguistics (Bybee 2010, Croft 2002, Langacker 1991). As outlined in the next section, Central Alaskan Yup'ik (CAY) has some 30 different sets of demonstratives, and they constitute a single formal category (Jacobson 1984a). ${ }^{1}$ In his staggering research on spatial reference, Levinson (1996, 2003) employs a feature of angular specification as a primary criterion for his hierarchical classification of spatial expression, and the demonstratives (i.e. deictic expressions) are grouped as spatial expressions working in the non-coordinate system (i.e. no angle specified); on the other hand, spatial concepts and their forms such as in front of or north of are classified as that functioning in the coordinate system (i.e. angle specified). Rukeyzer (2005) suggests that Levinson's framework on spatial typology may not work for the Eskimo demonstratives because they indicate some information on angular specification such as up or down as well as no inherent information on orientations such as this or that. For this reason, Rukeyzer (2005) proposes an alternative classification for the CAY demonstratives that does not rely on angular properties. While Rukeyzer's analysis has advanced the characterization of each Yup'ik demonstrative, however, it is still open to the questions of what characteristics Eskimo demonstratives share with those in other languages and of how unique they are when compared to those in other languages.

This article suggests that the property of angular specification that works well

$1 \quad$ All the data and basic observations on CAY are courtesy of Caan Toopetlook. I would also like to acknowledge the extensive works of Steven Jacobson as the primary source of my knowledge on CAY. The orthography of CAY employed in this article follows Jacobson (1995). 
Yuki-Shige Tamura

for typological descriptions of spatial expressions is still crucial for Eskimo spatial expressions, but the property makes a difference in Eskimo languages when it is applied as demonstrating gradience rather than dichotomy as shown in Levinson $(1996,2003)$. We argue that when the angular specification as a gradient concept is extended to the description of CAY demonstratives, it would illuminate a unique character of Eskimo demonstratives: the functions of Eskimo demonstratives are semantically stratified in terms of the degree of angular specification between degenerate cases like English this or that that do not constitute a frame of reference and highly specified cases that are close to typologically prototypical coordinate spatial expressions like north of or inside of. The gradience observed is theoretically characterized (i) with a semantic map (Croft 2001) that shows well how the demonstratives are stratified, and (ii) with subjectification (Langacker 1990), which motivates the continuation between spatial expressions prototypically described in the coordinate system and those in the non-coordinate system.

In what follows, basic features of the CAY demonstrative are briefly outlined in section 2, and the properties crucial to the discussion on angular specification are exemplified in section 3 with the review of Levinson's (2003) spatial typology. Furthermore, in section 4, one vital portion of demonstrative usage is characterized with the property of angular specification that illuminates a stratified relationship between the CAY demonstratives. Section 5 provides theoretical motivations for the analysis of section 4. Section 6 is for concluding remarks.

\section{Basic Characteristics of CAY demonstratives}

Jacobson (1984a, 1995:Ch.6) provides an excellent classification on how the CAY demonstratives are distributed in terms of spatial reference, employing the three parameters of (i) directivity, (ii) indicativity, and (iii) accessibility; this tripartite classification is now a standard tool for describing Eskimo demonstrative systems (Fortescue 1988). Table 1 below is a summary of the classification. The vertical column on the left indicates the classifications with the parameter of directivity, and the horizontal law is for the classifications of indicability, and the information on accessibility is attached under each category of directivity. 
Epistemicity and Deixis: Perspectives from Yup'ik

(1) Close to Speaker

(a) More accessible (near speaker)

(b) Less accessible (near listener)

extended restricted obscured

(2) Up from speaker

(a) More accessible (up the slope, same plane) paugna pingna pamna

(3) Away from speaker on level

man'a una imna

tamana tauna

pagna

pikna pakemna

(a) More accessible (over)
(b) Less accessible (across barrier)

(4) Down from speaker

(a) More accessible (down below, down the slope)

(b) Less accessible (toward exit, downriver)

augna

agna

un'a

unegna

(5) Inside/outside from speaker

(a) More accessible (inside)

(b) Less accessible (outside)

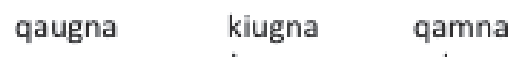

qagna keggna qakemna

(made based on Jacobsen (1984b:654, 1995:76) and Fortescue (1988:25)

Table 1. Central Alaskan Yup'ik's Demonstrative System

(3rd person singular, absolute case form employed)

To get a feel for how demonstratives work, let us consider examples from (2b) in the table. Suppose that a bird is up on a branch of a tree and you can see it, and you tell someone with you. In that case, you would say, "Atam (look!) pikna (that) yaqulek (bird)." The reason why pikna is chosen among the three demonstratives in the law is because the bird is not moving and is perceived in a single glance as a static object: it is regarded as restricted. If the bird is flying above you, you would employ the "extended" counterpart pagna rather than pikna: "Atam (look!) pagna (that) yaqulek (bird)," because the bird is moving, and you need a couple of glances to capture the trajectory. Moreover, if you can only hear a bird call from up in the tree, (i.e. you cannot see it), you would choose the "obscure" counterpart, and say "Atam (listen!) pakemna (that) yaqulek (bird)." One essential difference between (a) and (b) laws (i.e. accessibility) lies in whether or not you can reach the referent. In these cases, you cannot reach the bird or walk to it, and you are required to choose a demonstrative from (2b) rather than from (2a). Finally, the reason why (2) is chosen from the five categories (1) to (5) is because the speaker's neck is in an up position rather than just because the target is above the ground. For example, if the tree is further away and you could see the bird on the level without changing your neck position, you may employ a different demonstrative, ingna from (3a). By the same token, if the bird is sitting on the ground and it is close to you, you would describe it as kan'a (that) yaqulek (bird) 
Yuki-Shige Tamura

from (4a), because you are now looking down. However, if it is away and you can see it with your normal neck position, the bird is described as ingna yaqulek.

\section{Levinson's (2003) Typology of Spatial Expressions and the CAY Demonstrative System}

Now, while reviewing Levinson's (1996, 2003) typology on spatial expressions, let us further consider the characteristics of the CAY demonstrative system and discuss why the property of angular specification as a gradient concept is preferable to that of dichotomy. As mentioned in section 1, Levinson $(1996,2003)$ puts an emphasis on the parameter of angular specification for the typological classification of spatial expressions: whether or not an expression invokes a coordinate system when it is used. Examples (6) and (7) represent typical examples of spatial expressions in the non-coordinate system and in the coordinate system, respectively, and Levinson's accounts of the two groupings follow each set of the examples. Figure 1 below is an auxiliary diagram for (7) that is depicted following a Cognitive Grammar format (Langacker 1991), and the dotted arrow therein indicates that the speaker and the ground object for reference can be coincidental.

(6) Non-coordinate System Employed (angle not specified):
a. The orange is here.
(deictic)
b. The orange is in the bowl.
(contiguity)
c. John is at the office.
(named location)

[I]t is important to appreciate that deixis itself does not constitute a frame of reference. This is because deictic specifications of location merely use the deictic centre as a special kind of ground, and they do not themselves contribute to angular specification of the kind that constitute coordinate systems. (Levinson 2003:71)

(7) Coordinate System Employed (angle specified):
a. The statue by Giambologna is in front of the cathedral. (intrinsic)
b. The orange is to the left of the bowl.
(relative)
c. Amsterdam is north of Utrecht.
(absolute) 


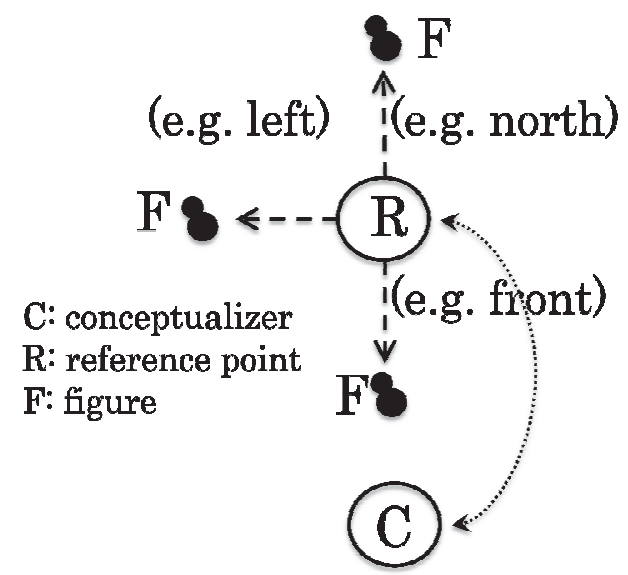

Figure 1. [The strategy is] to choose a prominent ground object at some remove from the figure or object to be located, and then to specify a search-domain from the ground by specifying an angle from that landmark,... (Levinson 1996:356)

Levinson's bilateral distinction for spatial expressions that results from the examinations of the spatial expressions of various languages highlights one unique characteristic of the CAY demonstrative: while most of the CAY demonstratives may show some angular specification, they are hardly employed for such cases of intrinsic, relative or absolute reference as in (7). In other words, the landmark that specifies an angle must be implicitly construed in exactly the same manner as the deictic center in the case of (6a). As mentioned in section 1, it is true, as Rukeyzer (2005) points out, that the CAY demonstratives shown in Table 1 should be considered as invoking some angular concepts except for (1: close to the speaker): (2: up from the speaker), (3: away from the speaker on level), (4: down from the speaker), and (5: insideloutside from the speaker). However, when CAY speakers employ a "prominent" ground object for the identification of a referent as in the coordinate cases like (7), they do not use a demonstrative, but choose a positional noun (Caan Toopetlook p.c.). Consider the examples below:

$$
\begin{aligned}
& \text { a. Estuulu-m aci-(ng)a iqa-uq. } \\
& \text { desk-erg space.under- 3rdposs dirty--ind. } 3^{\text {rd }} \text {.sing } \\
& \text { 'The space under the table is dirty.' } \\
& \text { b. Inar-ten aqui-gut elitnaurvi-im kete-nga-ni (ketiini) } \\
& \text { child-your play-ind.3rd.pl. school-erg front-3rdposs-loc } \\
& \text { 'Your children are playing in front of the school.' }
\end{aligned}
$$

As shown in examples (8), when a prominent ground object is invoked for a target reference like estuulu-m 'of desk' in (8a) or elitnaurvi-im 'of school' in (8b), CAY speakers choose a spatial expression from a set of positional nouns rather than from a set of demonstrative pronouns. (9) below is a partial list of the CAY positional nouns, and observe that same sort of spatial concepts as those in Table 1 are 
Yuki-Shige Tamura

also included in the set of positional nouns.

(9) Positional Nouns: (shown in 3rd person possessed form)

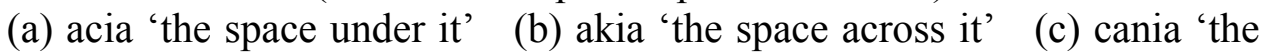
space beside it' (d) elatii 'the space outside it' (e) Ilua 'the space inside it' (f) qulii 'the space above it' (g) ketii 'the area in front of it (h) qainga 'its top' etc.

(see Jacobson 1995:99 for the whole list)

The following grammatical difference between the positional nouns and the demonstratives, furthermore, supports our semantic observation that the landmark that specifies an angle must be implicitly construed in the usage of the demonstrative pronouns: as shown in (8) and (9), positional nouns usually have to be realized in possessed form (Jacobson 1995:99) whereas "the demonstratives are not marked for possessor" (Jacobson 1984:653). In Eskimo languages, nominal inflection indicates information on case, person, number, and possessor (and its person and number). And the possessor itself is marked by ergative case as shown in (8). For example, the inflectional suffix of $-k a$, which indicates that the possessor is the first person singular, can attach to the root morpheme of (9f), and produces an acceptable form, qulka 'the space above me.' However, if it attaches to a demonstrative, say, the root morpheme of pikna (2b: up from the speaker), unacceptable forms like *pi'ka or *piknaka are created (Caan Toopetlook p.c.). This grammatical difference between qulka 'the space above me' and pikna 'up from the speaker' suggests that in contrast to coordinate spatial expressions exemplified in (7), the CAY demonstratives cannot conform their deictic center to a "prominent" landmark as shown in the dotted arrow in Figure 1. In sum, the CAY demonstratives preserve a crucial deictic nature: the point of reference should not be linguistically coded, even though they may invoke a coordinate system.

What Levinson $(1996,2003)$ does not put a focus on is the case in which coordinate systems may not always work together with an explicit reference point, which prevents us from characterizing Eskimo demonstratives beyond a mere aberration of the typological classification (cf. Levinson 2003:70). Our proposal, which suggests a stratified relationship should we dare to apply the notion of angular specification to the description of the CAY demonstrative system, is supported by the following two fundamental ideas of Cognitive Linguistics: (i) "it is important not to view the regularities as primary and the gradience and variation as secondary; rather the same factors operate to produce both regular patterns and the derivations," (Bybee 2010:6) and (ii) "the symbolic units of a language are heterogeneous, .... [T] heir distribution along these parameters is essentially continuous and does not offer any principled basis for dividing them into discrete components" (Langacker 1991:3). With these ideas and the observation above, we employ the property of angular specification as a gradient concept, and furthermore, the difference between coordinate and non-coordinate systems is supposed as continuous rather than distinct. 


\section{Epistemicity and Deixis: Perspectives from Yup'ik}

\section{A Stratified Relationship among the CAY Demonstratives}

When the CAY demonstrative system is analyzed in terms of the angle to a referent, we may find that some thirty CAY demonstratives are not evenly (or randomly) distributed as to the speaker's orientation to a target referent, but they can be arranged as those that narrow down the potential orientation of the speaker: it ranges from non-specified cases like English this or that that can be used in 360 degrees (i.e. no angle specified) to highly fixed cases that are close to the semantic configuration depicted in Figure 1 above. Table 2 below depicts the way in which the potential orientation to the referent gets specified gradually. Portions in gray are intended to show a potential location of a referent. In what follows, we exemplify each layer in order, and its implication is discussed in the next section. ${ }^{2}$

While the relationship between Layer I and II is shown in the next section, Layer I indicates the case of anaphoric use of a demonstrative. At this layer, the physical location of the referent does not matter, or has already been identified by the speaker and listener. For this function, CAY speakers utilize a demonstrative, imna (1a in Table 1), as shown in (10) below:

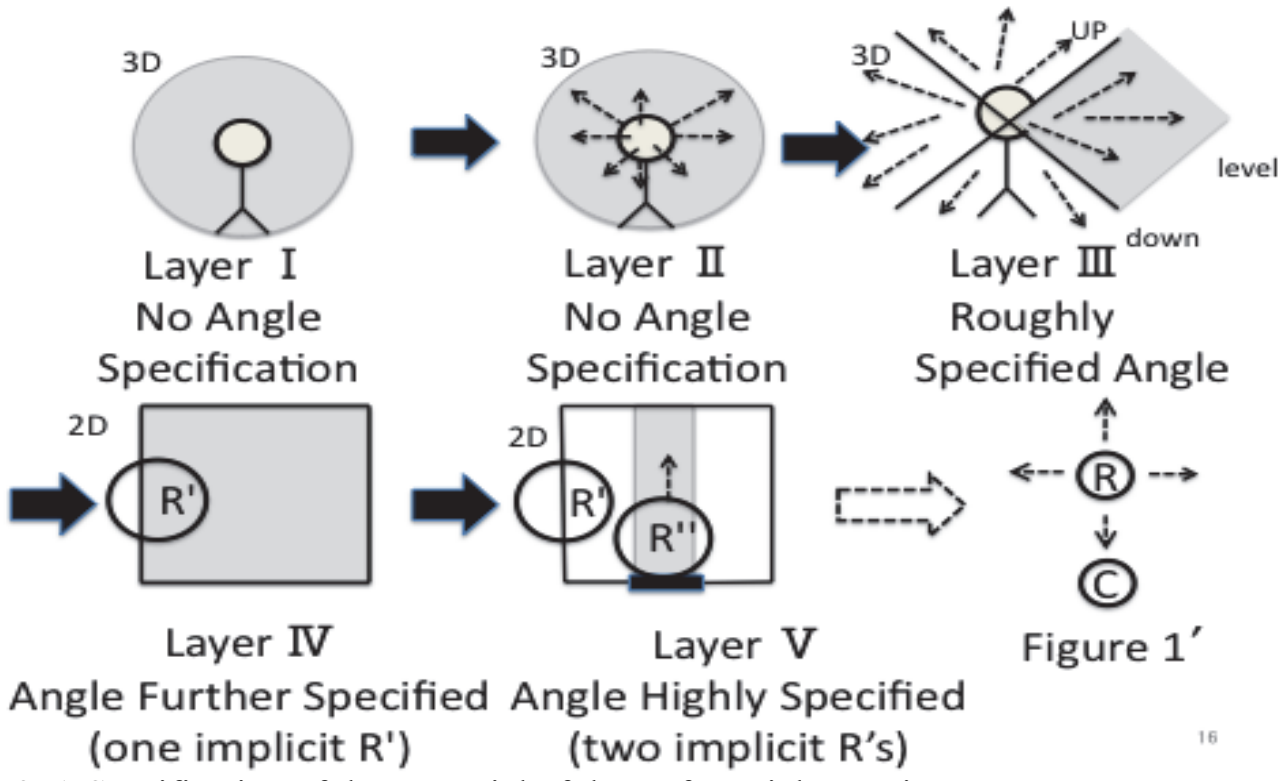

Table 2. A Stratification of the Potential of the Referential Function

(10) Payugte-llru-an=qaa

imna akuta-mek. give-past-ind.tran.2nd.sing $=\mathrm{Q}$ that.aforementioned ice cream-abl.mod 'Did you give that person Eskimo ice cream?'

$2 \quad$ Note that for the sake of a space constraint, the demonstratives in the column of "restricted" in Table 1 are essentially used as examples, but the same idea as to the speaker's orientation can be applied to the demonstratives in the other two subcategories. 
This usage shows one prototypical function of definiteness, and the reference point is considered as being implicitly placed at the speaker. ${ }^{3}$

Layer II indicates the stage where typologically prototypical functions of non-coordinate deictic expressions like (6a) are realized. The essence of this stage is that while the demonstratives of this layer may show an indicative function that accompanies some distance specification (e.g. proximal vs. distal), it does not limit the speaker's orientation (i.e. no angle specified). For this function, CAY speakers employ a demonstrative, una or tauna (1, in Table 1), depending on the distance from the speaker, as shown in (11):
(11) a. Una
calisuut-ngu-nrit-uq. .This isn't a tool.' this (near the speaker)
b. Tauna $=$ qaa tool-be-not-indi. ${ }^{\text {rd }}$.sing that (away from speaker) $=\mathrm{Q}$ hunting.tool-be- indi. $3^{\text {rd }}$.sing 'Is that one for hunting?'

Because the up-level-down distinction is introduced, the demonstratives working at Layer III are considered as being restricted in terms of angular specification compared to those in Layers I and II.

(12) a. Ingna that over.there bird
'That bird can't fly.'

b. Tangerr-sugna-an=qaa see-can-ind.tran.2sing $=\mathrm{Q}$ 'Can you see that airplane?'

c. Tugu-sciigat-aa pick.up-cannot-ind.tran.3sing that.down.below 'He can't pick up that fish (on the ground).'

As mentioned in section 2, the angle of the speaker's neck affects their choice of the demonstratives in this layer (see Jacobson 1984a). Though the diagram for this layer in Table 2 is for cases like (12a), ingna (3a in Table 1), of course, can be employed if the speaker turns around to refer to an object: it does not limit the speaker's orientation insofar as the speaker's neck is kept on the level. ${ }^{4}$

With the introduction of the implicit referential schema of enclosure, the demonstratives working at Layer IV are regarded as being more restricted in di-

3 Jacobson (1995) provides the following definition for imna: "the aforementioned one known to both speaker and listener', obscured, but not located in any place where it can be conveniently referred to on the basis of its location." (p.80)

$4 \quad$ If the speaker wants to refer to something behind him/her without turning around, the obscured counterpart amna (3a) will be employed for that purpose. The same observation can also be applied to pikna and kan'a, and the obscure counterparts, pakemna (2b) and camna (4a), are used in such a case, respectively. 


\section{Epistemicity and Deixis: Perspectives from Yup'ik}

rection than those at Layer III. As shown in the diagram for this layer, only the horizontal plane is concerned with the judgment of whether or not a referent is in an enclosure. In other words, while the search domain exercised at Layers II and III is three-dimensional, it becomes substantially two-dimensional at Layer IV. The opposition between the demonstratives at this layer is from (5a) and (5b) in Table 1. While anything can be invoked as an enclosure if CAY speakers regard it as so (Rukeyzer 2005), one typical instance of enclosure that is implicitly understood is a house, and it is natural to think, for instance, that examples (13a) and (13b) are uttered when the speaker is at the entrance door (Caan Toopetlook p.c.); both kiugna and keggna are restricted (i.e. visible).
(13) a. Kiugna
qavar-tuq
that.inside sleep.-ind.3rd 'That person is sleeping (in the house).'
b. Keggna
that.outside
qimugta nere-uq
neqe-mek.
'That dog is eating fish now outside.'

Finally, with the introduction of a point on the enclosure invoked at Layer IV, the criterion to employ a demonstrative becomes one-dimensional: the crucial factor in the usage of the demonstrative working at Layer $\mathrm{V}$ lies in whether a target referent is on a line or not. With the diagram for Layer $\mathrm{V}$ above, let us consider the following examples.
(14) a. Ugna
neqerrluk
Tegu-u.
that.near.exit dried.fish
pick.up-optative.
'Take that (near the door) dried fish.'
b. kiug-ku-t yuut sayuu-it-ut.
That.further.inside-ku-pl. Person-pl. tea-lack-ind.3pl.
'Those people (in the back of the room) don't have tea.'

When the enclosure invoked is a house, its exit serves as the point to create an implicitly construed line, (i.e. the straight line between the exit and the back). Jacobson (1995:80) provides for ugna and kiugna the following definitions respectively: "the one further toward the exit than the speaker" and "the one further in the house from the exit than the speaker." When these demonstratives are employed to refer to an object, the speaker's orientation will be highly fixed because the location of the target is limited on the line invoked. Though the same point is repeated, the enclosure and the point invoked on it must be implicit. For instance, if the door (amiik) is explicitly expressed (or perceived as the reference point like Figure 1), the positional noun ketii $(9 \mathrm{~g})$ should be utilized. ${ }^{5}$

$5 \quad$ This schematic configuration with the enclosure and point is also applied for an entire village to figure out the directions in the village: the river in the village corresponds to the straight line and its mouth the exit (Fortescue 1988). One important thing is that even in such an extended 
Yuki-Shige Tamura

\section{A Semantic Map for Demonstratives and its Theoretical Motivation}

The layers described above can be arranged as a semantic map (Croft 2002) as in Table 3 below. The arrows indicate how much languages develop their use of demonstratives as to the degree of angular specification. ${ }^{6}$ Let us now consider a typological implication and a theoretical motivation. Note that like English this or that, the Japanese demonstratives ( $a-, \mathrm{ko}^{-}$, and $\left.\mathrm{so}-\right)$ can show anaphoric and indicative uses, but they cannot be employed for the distinctions of Layers III to V.

\begin{tabular}{|c|l|l|l|l|l|l|}
\hline & $\begin{array}{l}\text { Prototypical Function } \\
\text { of Definiteness }\end{array}$ & & & & & $\begin{array}{l}\text { Prototypical Function of } \\
\text { Reference Point }\end{array}$ \\
\hline & Layer I & II & III & IV & V & VI (Figure 1' ) \\
\hline CAY & & & & & & \\
\hline English & & & & & & \\
\hline Japanese & & & & & & \\
\hline
\end{tabular}

Table 3. A Semantic Map for Demonstratives

The gradience is expressed in the semantic map that places a prototypical function of definiteness (epistemicity) and prototypical function of reference point at both ends. Layers I and II are realized by the demonstrative systems of a number of languages, and the relationship between I and II has been pointed out in the literature; for instance, the English definite article, the, has historically developed to the definite marker through the stages of I and II (e.g. Croft 2002:246). ${ }^{7}$ On the other hand, following Rijkhoff (2002), it is reported that some languages (e.g. Samoan) may strictly distinguish between layers I and II. They may group Layers II to VI together in contrast to the grouping of Eskimo languages. In addition, languages with the demonstrative system that develops to IV, or V may be described. What Table 3 suggests is that we can regard not as exceptional or deviant, but as a matter of degree, demonstrative systems that do not realize layers I and II as a single unit (i.e. only non-angular demonstratives) like English and Japanese.

Finally, the continuation between Layers I and VI may be motivated with the notion of subjectification developed by Langacker (1991). To grasp the idea, let us consider be going to as an example. While the phrase indicates a sense of future, Langacker (1991) suggests that the future sense is derived through the process in which the prominent part of the subject's physical motion observed in the original sense of going is semantically bleached away (i.e. subjectified) so that the implicit part of temporal progress inherent in the physical motion is left. Langacker (1991) argues that the phenomenon of semantic bleaching is ubiquitous in such a process

usage, a river or the mouth of a river is not utilized as an explicitly construed reference point like (7b) and (7c) (Caan Toopetlook p.c., and cf. Levinson 2003:109).

$6 \quad$ This map does not imply any historical development. I thank Eve Sweetser for the clarification at my presentation.

$7 \quad$ Rijkhoff (2002:92) also suggests that "the demonstrative has lost its deictic function and has in fact turned into a definite article." 


\section{Epistemicity and Deixis: Perspectives from Yup' 'ik}

as grammaticization or the formation of polysemous structure, synchronically and diachronically, examining prepositions, auxiliaries, and a number of grammatical constructions observed in English and other languages. The gradient relationship between Layers I to VI can also be regarded as reflecting the degree of subjectification, though the map is not intended to have any historical development. In the last section, we observed that the more the layer advances from I to V, the more complicated the deictic center, though implicitly construed, becomes in its schematic configuration. To put it the other way around, the configuration to restrict the angular specification gets simpler (i.e. bleached away) as the layer proceeds from VI to I.

\section{Concluding Remarks}

This article has suggested that while the property of angular specification for typological descriptions of spatial expressions is still crucial, it makes a difference in Eskimo languages when it is applied as showing gradience rather than dichotomy. We argued that when the angular specification as a gradient concept is extended to the description of CAY demonstratives, it clearly reveals a unique character: the functions of the Eskimo demonstratives are semantically stratified in terms of the degree of angular specification between non-specified cases that do not constitute a frame of reference and highly specified cases that are close to prototypical coordinate spatial expressions. The gradience observed is theoretically characterized with a semantic map and with subjectification, which motivates the continuation between spatial expressions prototypically described in the coordinate and non-coordinate systems. The importance of the property of angular specification as a typological parameter is further enhanced as we showed that it plays a crucial role in accounting for both of the systems.

\section{References}

Bybee, Joan L. 2010. Language, Usage and Cognition. Cambridge: Cambridge University Press.

Croft, William. 2002. Typology and Universals. Cambridge: Cambridge University Press.

Fortescue, Michael. 1988. Eskimo Orientation Systems. Meddelelserom Grønland, Man \& Society 11.

Jacobson, Steven A. 1984a. Semantics and Morphology of Demonstratives in Central Yup'ik Eskimo. Etudes/Inuit/Studies 8:185-192.

Jacobson, Steven A. 1984b. Yup'ik Eskimo Dictionary. Fairbanks: Alaska Native Language Center. 
Jacobson, Steve A. 1995. A Practical Grammar of the Central Alaskan Yup'ik Eskimo Language. Fairbanks: Alaska Native Language Center.

Langacker, Ronald W. 1991. Foundations of Cogntive Grammar vol. 2: Descriptive Appliation. Stanford : Stanford University Press.

Levinson, Stephen C. 1996. Language and Space. Annual Review of Anthropology 25:353-382.

Levinson, Stephen C. 2003. Space in Language and Cognition. Cambridge: Cambridge University Press.

Rijkhoff, J. 2002. The Noun Phrase. Oxford: Oxford University Press.

Rukeyser, Alison S. 2005. A Semantic Analysis of Yup'ik Spatial Deixis. PhD. thesis, University of California, Davis, Davis, CA.

Yuki-Shige Tamura

Shiga University

Faculty of Education

2-5-1 Hirastu, Otsu, Shiga,

Japan, 520-0862

tamura@edu.shiga-u.ac.jp 